\title{
EDITORIAL AND COMMENT What Should We Tell Our Patients About E-cigarettes?
}

\author{
Lori A. Bastian, $M D, M P H^{1,2}$ and Cheryl Oncken, $M D^{7}$ \\ 'Division of General Internal Medicine, University of Connecticut Health Center, Farmington, CT, USA; \\ ${ }^{2} V A$ Connecticut Healthcare System, Newington, CT, USA.
}

J Gen Intern Med 29(11):1427-8

DOI: $10.1007 / \mathrm{s} 11606-014-2943-5$

(c) Society of General Internal Medicine 2014

A $\mathrm{n}$ electronic cigarette (e-cigarette) is a battery-powered device that uses heat to vaporize a solution (often nicotine in propylene glycol and/or glycerol). E-cigarettes are popular, available in many flavors, and can be purchased in convenience stores and on-line. ${ }^{1}$ There are at least three categories of e-cigarette consumers: smokers who plan to continue smoking but want to use e-cigarettes when smoking is not allowed; smokers who plan to use the e-cigarette to assist them in quitting or cutting-down on smoking; and a minority of non-smokers who want to try something new (the concern is that this group will include minors). Although there are several public health concerns associated with e-cigarettes, we will highlight the issues most salient to the conversations general internists might have with their patients who smoke.

E-cigarettes have both positive and negative aspects. Available evidence suggests that e-cigarettes do not produce carbon monoxide, and may reduce a user's exposure to other toxicants, which in theory makes them safer than tobacco cigarettes. However, to date, there is no definitive evidence that e-cigarettes per se are safe., ${ }^{2,3}$ E-cigarettes have high satisfaction ratings, and this is attributed, in part, to the fact that inhaling the vapor delivers nicotine fast and mimics the sensory aspects of smoking. ${ }^{4}$ Studies have shown that using ecigarettes can reduce cravings and the desire to smoke. ${ }^{5}$

Although trials have shown that e-cigarettes can help smokers reduce their cigarette intake, the evidence is not sufficient to promote these products as a smoking cessation aid. ${ }^{2,3,6}$ A lack of efficacy in clinical trials, along with examinations of patterns of use in surveys, ${ }^{7}$ raises the concern that many smokers will become dual users of both tobacco cigarettes and e-cigarettes, of which the long-term health effects are unknown. There is also concern about secondhand exposure to the chemical constituents in the vapor from e-cigarettes. Although e-cigarettes appear to be an efficient delivery system for nicotine, there is variability of how much nicotine is delivered in different ecigarette brands. ${ }^{8}$ At this time, the US Food and Drug

Published online July 9, 2014
Administration (FDA) has not finalized its regulatory process for e-cigarettes.

In this issue of JGIM, Steinberg and colleagues report on a cross-over trial comparing the e-cigarette with the FDAapproved nicotine replacement inhaler in terms of perceived benefits, harms, appeal, and role in assisting with smoking cessation. ${ }^{9}$ Briefly, the study had complete data on 38 current smokers age 18 and older with no prior experience using the e-cigarette or the nicotine inhaler. After using each product for 3 days, in random order, with a washout period in between, the e-cigarette had a significantly higher total satisfaction score and higher reward score than the nicotine inhaler. More subjects reported they would use the e-cigarette to make a quit attempt $(76 \%)$ than the inhaler (24\%). Overall, the e-cigarette was more acceptable, provided more satisfaction, and had higher perceived benefit than the nicotine inhaler during this trial. This study provides more evidence of the acceptability and perceived benefits of e-cigarettes.

Patients are frequently asking their General Internists about e-cigarettes. ${ }^{4}$ How should internists handle the concern about recommending a product that is not regulated by the FDA and which lacks evidence on safety and efficacy? Internists are in a unique position to educate their patients about these products.

Here is a recent dialogue in clinic between a General Internist and her patient.

Patient: I quit smoking for 2 months last year. I am planning to quit smoking again on my birthday.

Internist: That is great. Learning from your experience with smoking cessation last year, how can I help you this year? Do you want a referral to the smoking cessation clinic? What about trying a telephone quitline and a medication to help you quit? Patient: No, I don't want a referral. This time I am going to use the e-cigarette to help me quit.

Internist: What makes you want to try an ecigarette?

Patient: E-cigarettes are popular and my sister-inlaw used the e-cigarette and it helped her quit. Do you think e-cigarettes are safe?

Internist: I am not sure. I have read studies that show variations in the amount of nicotine delivered 
in the vapor and I want to know how much nicotine you are getting. My concern is that you will continue to smoke tobacco cigarettes and also use e-cigarettes. We don't know the long-term health effects of e-cigarettes.

Patient: Don't worry Doc. I actually like the dirty taste of tobacco and once I get over my nicotine addiction, I will stop using the e-cigarette.

Internist: Please be careful. I prefer that you use one of the over-the-counter nicotine replacement products or prescription medications for smoking cessation because they are regulated by the FDA. I am proud of you for wanting to quit smoking and I want to do what I can to support your plan.

This actual conversation highlights some of the issues facing general internists and their patients; 1) some patients want to use e-cigarettes as cessation aids; and 2) physicians want to be supportive of their patients' attempts to quit smoking but concerns exist regarding safety and efficacy of e-cigarettes, as well as dual use of e-cigarettes and tobacco cigarettes. Steinberg and colleagues have suggested that e-cigarettes have the potential to be important nicotine delivery products owing to their high acceptance, but they also point out that more data are needed to evaluate their efficacy and safety. ${ }^{9}$ Future studies should explore reasons why the e-cigarette is perceived to be better than FDAapproved smoking cessation medications. Is this because of marketing or is this because the mechanism mimics the sensory aspects of smoking? Are e-cigarettes socially more acceptable? Do e-cigarettes' flavors have a particular appeal to smokers?

In summary, e-cigarettes might be safer than tobacco cigarettes because they do not burn tobacco and they do not produce carbon monoxide. On the other hand, there may be long-term health effects associated with the product that will not be known for years. E-cigarettes may hold value as a harm reduction strategy among those unwilling or unable to quit. However, more data are needed on the following: 1) evidence on how much nicotine various brands of e-cigarettes deliver; 2) evidence on safety of the other chemicals delivered by e-cigarettes; 3) evidence on how many people who start e-cigarettes as a cessation aide continue to use both cigarettes and e-cigarettes; and 4) evidence on whether e-cigarettes actually help people to quit smoking. This research on efficacy and long-term safety would allow physicians to more appropriately inform their patients about these products.

Corresponding Author: Lori A. Bastian, MD, MPH; Division of General Internal Medicine, University of Connecticut Health Center, 263 Farmington Ave, Farmington, CT 06030, USA (e-mail: bastian@uchc.edu).

\section{REFERENCES}

1. King BA, Alam S, Promoff G, Arrazola R, Dube SR. Awareness and everuse of electronic cigarettes among US Adults, 2010-2011. Nicotine Tob Res. 2013;15:1623-7.

2. Grana R, Benowitz N, Glantz SA. E-cigarettes: a scientific review. Circulation. 2014;129:1972-86.

3. Etter JF, Bullen C, Flouris AD, Laugesen M, Eissenberg T. Electronic nicotine delivery systems: a research agenda. Tob Control. 2011;20:243-8.

4. Pepper JK, Brewer NT. Electronic nicotine delivery system (electronic cigarette) awareness, use, reactions and beliefs: a systematic review. Tob Control. 2013. doi:10.1136/tobaccocontrol-2013-051122.

5. Vansickel AR, Eissenberg T. Electronic cigarettes: effective nicotine delivery after acute administration. Nicotine Tob Res. 2013;15:267-70.

6. Bullen C, Howe C, Laugesen M, McRobbie H, Parag V, Williman J, Walker N. Electronic cigarettes for smoking cessations: a randomized controlled trial. Lancet. 2013;382:1629-37.

7. Lee YO, Hebert CJ, Nonnemaker JM, Kim AE. Multiple tobacco product use among adults in the United States: cigarettes, cigars, electronic cigarettes, hookah, smokeless tobacco, and snus. Prev Med. 2014;62:14-9.

8. Goniewicz ML, Hajek P, McRobbie H. Nicotine content of electronic cigarettes, its release in vapour and its consistency across batches: regulatory implications. Addiction. 2014;109:500-7.

9. Steinberg MB, Zimmermann MH, Delnevo CD, Lewis MJ, Shukla P, Coups EJ, Foulds J. E-cigarette vs. nicotine inhaler: comparing the perceptions and experiences of inhaled nicotine. JGIM. 2014. doi:10.1007/s11606-014-2889-7. 